\title{
O APRIMORAMENTO DO MÉTODO SEGUNDO OS PRINCÍPIOS DE KAIZEN E SUAS LIMITAÇÕES
}

\author{
Gilberto Tavares de Macedo \\ Dias \\ gilbertotmd@id.uff.br \\ Programa de Pós-graduação \\ em Dinâmica dos Oceanos e da \\ Terra, Instituto de Geociências, \\ Universidade Federal Fluminense - \\ UFF, Niterói, Rio de Janeiro, Brasil
}

\section{Mirian Araújo Carlos Crapez}

miriancrapez@id.uff.br

Programa de Pós-graduação em Dinâmica dos Oceanos e da Terra, Instituto de Geociências, Universidade Federal Fluminense UFF, Niterói, Rio de Janeiro, Brasil

\section{Nuno Leal}

nunocunhasilva@gmail.com Alevinus Biotecnologia e Inovação, Rio de Janeiro, Rio de Janeiro, Brasil

\section{Christina Machado}

publiomcm@gmail.com

Programa de Pós-graduação em Dinâmica dos Oceanos e da Terra, Instituto de Geociências, Universidade Federal Fluminense UFF, Niterói, Rio de Janeiro, Brasil

Estefan Monteiro da Fonseca oceano25@hotmail.com Programa de Pós-graduação em Dinâmica dos Oceanos e da Terra, Instituto de Geociências, Universidade Federal Fluminense UFF, Niterói, Rio de Janeiro, Brasil
Após a guerra, no Japão da década de 1950, um conjunto de procedimentos passou a ser adotado pelos empresários da época em busca de melhores resultados e maior foco no trabalhador. Neste momento surge o método Kaizen cujo significado é "mudança para melhor". Esta filosofia não se limitou apenas ao campo de trabalho, mas também passou a ser hábito familiar e pessoal.

Esta filosofia preconiza a melhoria da capacidade dos indivíduos, progressivamente. Segundo este método, o caminho mais adequado seria através de um objetivo comum e da predominância da coletividade sobre o indivíduo, sempre com foco no ser humano. Nesta linha, a colaboração e o aperfeiçoamento da comunidade, em conjunto com outras medidas de sustentabilidade, as quais visam reduzir desperdícios e custos, é o que garante o sucesso na produtividade e na qualidade do produto ofertado (Vivan et al., 2016).

Por outro lado, a filosofia de Kaisen não se mostra totalmente eficaz se não considerado o conceito de entropia organizacional, conceito este, segundo o qual, em todo processo de transformação de energia em trabalho produtivo, uma fração da energia será desperdiçada em decorrência da fase de organização (Rocha et al., 2011). Sendo assim, não pode existir máquina $100 \%$ eficiente porque a entropia não permite. Se entendermos o ser humano como parte da engrenagem produtiva, a entropia organizacional estaria principalmente na necessidade de mudança cultural. Prativedwannakij (2008) avaliou que os problemas no desenvolvimento de atividades Kaizen na Tailândia vieram tanto da administração quanto de sua equipe. Posteriormente, Prativedwannakij (2009) discute que questões como falta de comprometimento na conclusão de planos e a falta de competitividade entre os trabalhadores prejudica a implementação dos princípios de melhoria da produção. Com base nestas informações, pressupõe-se que a extensão da filosofia de Kaizen para diferentes povos nos obriga considerar a entropia organizacional no polinômio da produtividade (Recht; Wilderom, 2017).

Atualmente, como forma de otimização de gastos de produção, ferramentas como o Life Cycle Assessment (LCA), ou, em português, "Avaliação do Ciclo de Vida", são usadas para identificar os riscos ambientais de produtos, serviços e processos de fabricação existentes e planejados, bem como identificar oportunidades de melhoria estratégica (Guinnée et al., 2011). Por outro lado, o uso de ferramentas sociais deve ser considerado para a minimização da entropia organizacional. Entre estas ferramentas está a atuação de stakeholders que percolam a estrutura da sociedade tornando mais efetiva a neutralização das reações negativas à mudança de cultura (Sartury et al., 2015). 
Não obstante, questões relativas à adequação de produtividade à sustentabilidade ambiental também se encaixam no contexto descrito no presente texto. Reações contrárias à adaptação da atual indústria à realidade de sustentabilidade ecológica se apresentam constantes, uma vez que também estas transformações se apresentam no campo cultural.

Assim, com base nos conceitos supraexpostos, fica evidente que a produtividade humana nos diversos setores necessários para a sua subsistência não consiste em um processo simples. De forma contrária, a produtividade depende da sinergia de diversos fatores que devem ser considerados em profundidade e em conjunto, como as células constituintes de um ser vivo. Suas respectivas importâncias nos processos "bioquímicos" não se limitam ao seu tamanho aparente, mas ao peso de suas atribuições "fisiológicas". O que a história tem apresentado aos mais atentos, é que com a evolução das sociedades e relações produtivas, o aprimoramento dos métodos através de procedimentos holísticos se torna cada vez mais importante na sobrevivência deste "biofilme" que denominamos humanidade.

\section{REFERÊNCIAS}

Guinée, J. B., Heijungs, R., Huppes, G. et al. 2011. Life Cycle Assessment: Past, Present, and Future. Environmental Science \& Technology 45, 1:90-96. https://doi.org/10.1021/es101316v

Prativedwannakij, K. 2008. "Kaizen" Development in Thailand's industries: A Comparative Study between Japan and Thailand. Doctoral thesis, Graduate School of Asia and Pacific Studies, WASEDA University.

Prativedwannakij, K. 2009. The Factors of Thai Workers' Behavioral Characteristics during Kaizen Activities. Kasetsart Journal: Social Sciences 30, 3:313-322.

Recht, R., Wilderom, C. 1998. Kaizen and culture: on the transferability of Japanese suggestion systems. International Business Review 7, 1:7-22.

Rocha, I., Hein, N., Lavarda, C. E. F., Nascimento, S. 2011. A presença da entropia da informação no controle orçamentário em ambiente inovador. Revista de Administração e Inovação 8, 2:82-105.

Sarturi, G., Seravalli, C. Boaventura, J. M. G. 2015. Afinal, o que é distribuir valor para os stakeholders? Uma análise bibliográfica sobre o tema. Revista de Administração da UFSM 8, 92-113. https://doi.org/10.5902/1983465916556

Vivan, A. L., Ortiz, F. A. H., Paliari, J. C. 2016. Modelo para o desenvolvimento de projetos kaizen para a indústria da construção civil. Gestão \& Produção 23, 2:333-349. https://doi. org/10.1590/0104-530×2102-15 